\title{
SECAGEM CONVECTIVA DE YACON: INFLUÊNCIA DO PRÉ-TRATAMENTO OSMÓTICO E DA TEMPERATURA DE SECAGEM
}

\author{
F. J. LOPES ${ }^{1 *}$, R. E. MELLO JR ${ }^{1}$, A.P.L. SILVEIRA ${ }^{1}$, G. E. ALVES ${ }^{1}$, J. R. J. JUNQUEIRA ${ }^{1}$, J. L. \\ G. CORREAA ${ }^{1}$ \\ ${ }^{1}$ Universidade Federal de Lavras, Departamento de Ciências dos Alimentos \\ *e-mail:francemirlopes@yahoo.com.br
}

\begin{abstract}
RESUMO
O yacon tem como principal carboidrato de reserva os frutooligossacarídeos, os quais têm sido recomendados em dietas para pessoas diabéticas e obesas. Porém, quando destinado ao consumo, deve ser processado ou consumido rapidamente. $\mathrm{O}$ objetivo deste trabalho foi estudar a cinética de secagem convectiva de fatias de yacon, avaliando as influências da temperatura e do uso de desidratação osmótica na difusividade e tempo de secagem. A desidratação osmótica com pulso de vácuo foi realizada com solução de sorbitol, $38^{\circ}$ Brix a $35^{\circ} \mathrm{C}$ e de $74 \mathrm{mmHg}$ de pulso de vácuo nos primeiros 10 minutos da desidratação. Foram testadas temperaturas de secagem de $40 ; 50 ; 60$ e $70^{\circ} \mathrm{C}$ e velocidade do ar constante em $0,5 \mathrm{~m} / \mathrm{s}$. A cinética de secagem foi ajustada pelo modelo de Fick e a relação da difusividade com a temperatura pela equação de Arrhenius. Os resultados mostraram que a cinética de secagem do yacon é fortemente influenciada pela temperatura e também pela PVOD, com menor tempo de processo e maior difusividade efetiva em temperaturas mais elevadas e/ou quando a amostra é pré-tratada. Os modelos de Fick e Arrhenius apresentaram bom ajuste aos dados experimentais de cinética de secagem e variação de difusividade com a temperatura.
\end{abstract}

\section{INTRODUÇÃO}

A secagem é a operação unitária na qual ocorre eliminação de água por transporte simultâneo de calor e massa. É, provavelmente, o mais antigo método de conservação de alimentos. (PARK; LAMSAL; BALASUBRAMANIAM, 2014).

A secagem tem como objetivo promover a redução da atividade de água, proporcionando inibição do crescimento microbiano, diminuição da atividade enzimática e velocidades das reações químicas. Porém, a exposição do produto a temperaturas elevadas por longos períodos pode causar perdas de qualidade nutricionais e sensoriais. A desidratação osmótica seja a pressão atmosférica ou com uso de pulso de vácuo (PVOD- pulsed vacuum osmotic dehydration) costuma auxiliar na minimização de perda de qualidade e levar a redução de tempo de secagem (FANTE et al., 2011; CORRÊA et al., 2014).

$\mathrm{Na}$ maioria dos processos osmóticos, são utilizadas soluções de sacarose (CORRÊA et al., 2010; ZOU et al., 2013). Porém, agentes como o sorbitol são favoráveis para a obtenção de alimentos para pacientes com restrições a aqueles produtos, como diabéticos e hipertensos. O sorbitol é um eficiente agente desidratante, pois proporciona uma alta razão de perda de água por ganho de sólidos (CHAUHAN et al., 2011). 
A modelagem da cinética de secagem pode ser feita com o uso de modelos difusivos baseados na teoria da difusão de Fick (CRANK, 1975). Embora este modelo apresente deficiências como desconsiderar a resistência externa e não considerar o encolhimento do produto durante a secagem, o ajuste proporcionado pelo mesmo é bastante satisfatório e a difusividade obtida pelo ajuste auxilia na interpretação do processo de secagem (WALKER, L.; SENADEERA, W, 2014).

O presente trabalho teve como objetivo estudar a cinética de secagem convectiva de fatias de yacon, avaliando a influência da temperatura e do uso de PVOD na difusividade e tempo de secagem.

\section{MATERIAL E METODOS}

\subsection{Material}

Tubérculos de yacon (Smallanthus sonchifolius) foram obtidos junto ao comércio local de Lavras, Minas Gerais. Para seleção da matéria-prima foram considerados o grau de maturação, tamanho, peso, intensidade de cor e firmeza uniformes. Além disso, a ausência de doenças, fissuras e outras injúrias físicas foram levadas em consideração.

Os tubérculos foram utilizados nos experimentos logo após sua aquisição.

\subsection{Preparo das amostras e da solução osmótica}

As raízes selecionadas foram lavadas em água corrente e descascados manualmente com uma faca de aço inox. As amostras foram cortadas em dimensões de 2,00 x 2,00 x 0,50 $\mathrm{cm}$ (comprimento $\mathrm{x}$ largura $\mathrm{x}$ espessura) utilizando-se um fatiador manual. As dimensões das fatias foram aferidas com um paquímetro digital (Western, 150mm-DC-60, China).

A solução osmótica foi preparada com água destilada e sorbitol comercial (Singsino
Goup Limited, China) em concentração de $38^{\circ}$ Brix.

\subsection{Condições experimentais}

As amostras de yacon foram divididas em dois conjuntos. Em um deles, foi aplicado o tratamento osmótico anteriormente a secagem e o outro foi submetido à secagem sem pré-tratamento.

2.3.1 Desidratação osmótica assistida por pulsos de vácuo (PVOD)

As amostras foram acondicionadas em erlenmeyers contendo solução osmótica de sorbitol na proporção massa de yacon e massa de solução de 1:10 (w/w). A desidratação osmótica foi realizada a temperatura de $35^{\circ} \mathrm{C}$ e pressão de vácuo de $74 \mathrm{mmHg}$ nos primeiro $10 \mathrm{~min}$ processo, com tempo total de $300 \mathrm{~min}$. Estas condições foram otimizadas em trabalho anterior (OLIVEIRA, 2013), por proporcionarem maior retenção de frutanos.

Após a PVOD, as amostras foram retiradas dos erlenmeyers e imediatamente imersas em um banho de gelo por 10 segundos. A água de lavagem superficial foi removida por secagem da superfície com papel toalha. Este procedimento tem o objetivo de cessar a desidratação e retirar o excesso de solução desidratante da superfície da amostra (CORRÊA et., 2014).

As amostras foram acondicionadas em potes herméticos para posterior secagem. Parte das amostras foi submetida a determinação de teor de umidade para se avaliar a perda do teor de umidade no processamento osmótico.

\subsubsection{Secagem}

A secagem das amostras foi realizada num secador convectivo a $40 ; 50 ; 60$ e $70^{\circ} \mathrm{C}$ e velocidade constante de $0,5 \mathrm{~m} \mathrm{~s}^{-1}$ até teor de umidade final de $13 \mathrm{~kg}$ água/100 kg material seco. A massa das amostras foi medida em tempos pré-definidos para obtenção da cinética de secagem. A secagem foi mantida 
até peso constante para a determinação do teor de umidade de equilíbrio.

\subsection{Análises}

2.4.1. Determinação do teor de umidade

O teor de umidade foi determinado segundo método gravimétrico 934.06 da (AOAC, 2005) em estufa a vácuo (pressão $\leq$ $100 \mathrm{mmHg}$ ) a $70^{\circ} \mathrm{C}$ até peso constante.

\subsubsection{Atividade de água}

A determinação da atividade de água foi realizada à temperatura de $25^{\circ} \mathrm{C}$ com o uso de um medidor de atividade de água (Aqualab Decagon Devices Inc. Pullman, modelo CX2T, Washington, EUA).

\subsection{Modelagem da cinética de secagem}

O modelo utilizado nos ajustes da cinética de secagem do yacon baseia-se na equação de difusão de unidirecional Fick (CRANK, 1975):

$$
\frac{\partial M_{(t)}}{\partial t}=\frac{\partial}{\partial z}\left(D_{e f f} \frac{\partial M_{(t)}}{\partial z}\right)
$$

onde $\mathrm{M}(\mathrm{t})$ é a quantidade de água ou de sólidos no instante $\mathrm{t}$, é $\mathrm{D}_{\text {eff }}$ é a difusividade efetiva e z é a direção da transferência.

O modelo de difusividade efetiva de Fick relaciona a variação do teor de umidade com o tempo e dimensões, de acordo com a lei de difusão de Fick pode ser resolvido considerando placa semi-infinito com espessura 2L, teor de umidade inicial uniforme, $M_{(z, 0)}=M_{0}$; a concentração de simetria, $\left.\frac{\partial M(t)}{\partial t}\right|_{z=0}=0$; e o teor de umidade de equilíbrio na superfície do material, $M_{(L, t)}=M_{e q}$; Como resultado, a equação de difusão de Fick unidirecional (CRANK, 1975) torna-se:

$$
M R=\frac{8}{\pi} \sum_{n=0}^{\infty} \frac{1}{(2 n+1)^{2}} \exp \left[-(2 n+1)^{2} \frac{\pi^{2}}{4} \frac{d_{e f f} t}{L^{2}}\right]
$$

onde $\mathrm{D}_{\text {eff }}$ é a difusividade efetiva de água, L é metade da espessura da amostra, $n$ é o número de termos, MR é a razão de umidade, t é o tempo de secagem.

A razão de umidade (MR) foi definida como:

$M R=\frac{\left(M_{t}-M_{e}\right)}{\left(M_{0}-M_{e}\right)}$

onde $\mathrm{M}_{\mathrm{t}}$ é o teor de umidade do yacon em cada momento, $\mathrm{M}_{0}$ teor de umidade inicial do yacon e $\mathrm{M}_{\mathrm{e}}$ o teor de umidade de equilíbrio.

A dependência de $D_{\text {eff }}$ com a temperatura foi analisada pela equação de Arrhenius:

$D_{\text {eff }}=D_{0} \exp \left(-\frac{E_{a}}{R T}\right)$

onde $\mathrm{D}_{0}$ é o fator pré-exponencial da equação Arrhenius, $\mathrm{E}_{\mathrm{a}}$ a energia de ativação $\left(\mathrm{kJ} \mathrm{mol}^{-1}\right)$, $\mathrm{R}$ a constante universal dos gases, 8,314 $\mathrm{J}$ $\mathrm{mol}^{-1} \mathrm{~K}^{-1}$ e a temperatura absoluta $(\mathrm{K})$.

Os ajustes dos modelos de cinética de secagem e de dependência da difusividade com relação à temperatura aos dados experimentais foram obtidos com auxílio do suplemento Solver do software Excel.

\section{RESULTADOS E DISCUSSÃO}

\subsection{Desidratação osmótica com pulso de vácuo (PVOD)}


A PVOD do yacon resultou na redução de teor de umidade inicial de $8,58 \pm 0,37 \mathrm{~kg}$ água/ $\mathrm{kg}$ material seco para 2,44 $\pm 0,19 \mathrm{~kg}$ água/ kg material seco. Observa-se que houve também redução da atividade de água de $0,989 \pm 0,001$ para $0,976 \pm 0,004$, perda de água de $51,23 \%$ e ganho de sólido de $5,87 \%$. Estes resultados estão de acordo com os dados obtidos por Oliveira (2013).

\subsection{Secagem}

As Figuras 1 e 2 apresentam as curvas das cinéticas de secagem do yacon com e sem pré-tratamento por PVOD, respectivamente, em função da razão de umidade (MR) e a Tabela 1 apresenta os tempos de secagem.

Figura 1. Cinética de secagem de yacon desidratados em PVOD

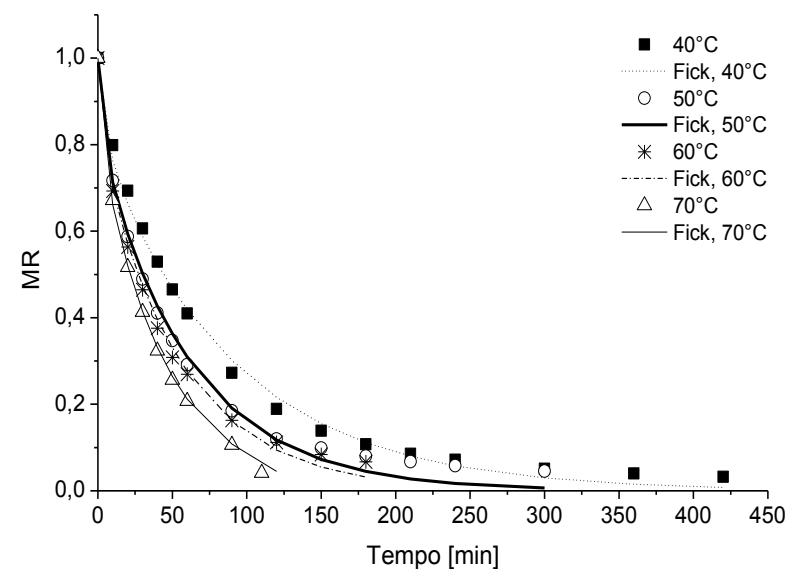

Figura 2. Cinética de secagem de yacon

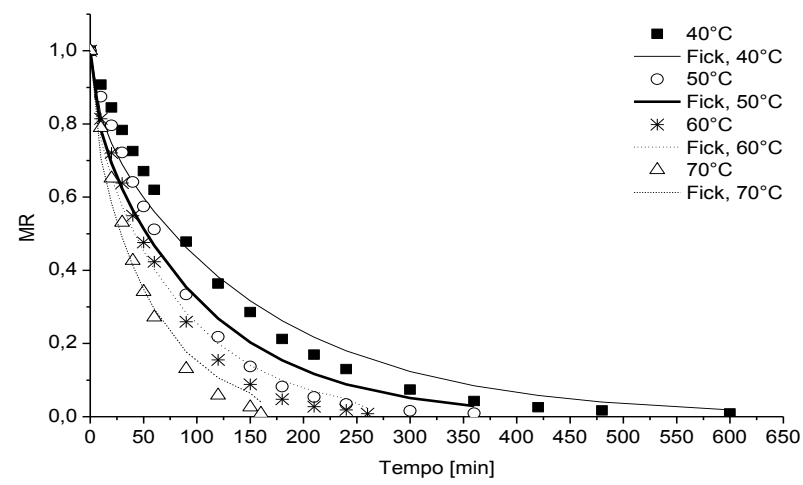

Observa-se a temperatura levou a redução nos tempos de secagem, independentemente do uso de pré-tratamento. Adicionalmente, em todas as temperaturas, a razão de umidade reduziu mais rapidamente no início da secagem, com redução mais acentuada em maiores temperaturas. A influência da temperatura é bem relatada (DJENDOUBI MRAD et al., 2012; GIRI; PRASAD, 2007; VEGA-GÁLVEZ et al., 2012). O aumento de temperatura resulta em maior excitação de moléculas de água no interior do material com consequente maior difusividade e pressão de vapor mais elevada (SADEGHI et al., 2013).

A utilização da PVOD como prétratamento para a secagem convectiva proporcionou a redução do tempo de secagem quando comparadas com as amostras que não sofreram o pré-tratamento (Figura 1 e 2, Tabela 1), independente da temperatura. Embora o teor de frutanos não tenha sido medido neste trabalho, observa-se que a PVOD pode ter auxiliado na sua retenção, uma vez que o composto é termossensível e uma menor exposição períodos prolongados a temperaturas é interessante para este tipo de composto (SCHER; RIOS; NOREÑA, 2009).

\subsection{Modelagem da cinética}

$\mathrm{Na}$ Tabela 1, Figura 1 e 2 são apresentados os resultados dos ajustes do modelo de Fick aos dados experimentais. Pode-se observar que, em termos do coeficiente de determinação, o modelo de Fick representa bem a cinética de secagem do yacon, em todos os casos $\mathrm{R}^{2}>0,98$ e variância acima de $97 \%$.

Os valores da difusividade efetiva $\left(D_{\text {eff }}\right)$ corroboraram o fenômeno da secagem, uma vez que apresentaram valores maiores para experimentos em que se utilizou a PVOD como pré-tratamento e em experimentos em que se utilizou maiores temperaturas (Tabela $1)$. 
Os valores de $\mathrm{D}_{\text {eff }}$ estão de acordo com DOYMAZ (2008), 1,42 × $10^{-9} \mathrm{~m}^{2} \mathrm{~s}^{-1}$ para morangos e NOWACKA et al (2012), 1,89 a $2,16 \times 10^{-9} \mathrm{~m}^{2} \mathrm{~s}^{-1}$ para amostras de maça seca pré-tratadas. Resultados diferentes aos relatados por DOYMAZ (2012), 7,05 x10 ${ }^{-11} \mathrm{e}$ $2,34 \times 10^{-10} \mathrm{~m}^{2} \mathrm{~s}^{-1}$ para a secagem de caquis, e pela GUINÉ et al., (2011), 4,08 x $10^{-8}$ e $2,2 \mathrm{x}$ $10^{-7} \mathrm{~m}^{2} \mathrm{~s}^{-1}$ na secagem convectiva de abóbora em 30 e $70^{\circ} \mathrm{C}$. A difusividade varia de acordo com características da amostra, como variedade, composição, geometria, temperatura do ar e pré-tratamento, o que explica algumas das diferenças encontradas nos resultados em comparação com alguns relatados na literatura.

Tabela 1 - Tempo de secagem, Coeficiente de difusividade efetiva $\left(D_{\text {eff }}\right)$, coeficiente de determinação $\left(R^{2}\right)$, raiz do erro quadrático médio (RMSE) e Variância (\%Var) do modelo de Fick para as diferentes temperaturas estudadas.

\begin{tabular}{cccccc}
\hline & Tempo de secagem & & & \\
Tratamento & {$[\mathrm{min}]$} & $\mathrm{D}_{\text {eff }} \times 10^{9}\left[\mathrm{~m}^{2} \mathrm{~s}^{-1}\right]$ & $\mathrm{R}^{2}$ & RSME & $\%$ Var \\
\hline $\mathrm{CP} 40^{\circ} \mathrm{C}$ & 420 & 0,8415 & 0,9968 & 0,017 & 99,58 \\
$\mathrm{CP} 50^{\circ} \mathrm{C}$ & 300 & 1,2278 & 0,9967 & 0,022 & 99,25 \\
$\mathrm{CP} 60^{\circ} \mathrm{C}$ & 180 & 1,3709 & 0,9971 & 0,020 & 99,34 \\
$\mathrm{CP} 70^{\circ} \mathrm{C}$ & 110 & 1,7103 & 0,9991 & 0,010 & 99,82 \\
$\mathrm{SP} 40^{\circ} \mathrm{C}$ & 600 & 0,4789 & 0,9894 & 0,041 & 97,97 \\
$\mathrm{SP} 50^{\circ} \mathrm{C}$ & 360 & 0,7044 & 0,9855 & 0,042 & 97,60 \\
$\mathrm{SP} 60^{\circ} \mathrm{C}$ & 260 & 0,8927 & 0,9924 & 0,027 & 98,85 \\
$\mathrm{SP} 70^{\circ} \mathrm{C}$ & 160 & 1,2910 & 0,9920 & 0,024 & 98,88 \\
\hline
\end{tabular}

Onde CP significa secagem com pré-tratamento por PVOD e SP, secagem sem PVOD

A tabela 2 apresenta os valores do fator pré-exponencial $\left(\mathrm{D}_{\mathrm{o}}\right)$ e energia de ativação $\left(E_{a}\right)$ obtidos pelo ajuste de $D_{\text {eff }}$ em função da temperatura pela equação de Arrhenius. Observou-se que o ajuste foi bastante satisfatório, com $\mathrm{R}^{2}$ maiores que 0,95. Resultados semelhantes aos encontrados por TAHERI-GARAVAND et al., (2011) Além disso, os valores de $\mathrm{E}_{\mathrm{a}}$ estão próximos ao encontrados por DOYMAZ et al., (2014) e se dentro da faixa de 12,7 a $110 \mathrm{~kJ} \mathrm{~mol}^{-1}$ definida por ZOGZAS et al., (1996) para produtos alimentícios.

Tabela 2 - Valores referentes a energia de ativação e fator pré-exponencial da equação de Arrhenius

\begin{tabular}{ccccc}
\hline Trat. & $\mathrm{E}_{\mathrm{a}}\left[\mathrm{kJ} \mathrm{mol}^{-1}\right]$ & $\mathrm{D}_{0}\left[\mathrm{~m}^{2} \mathrm{~s}^{-1}\right]$ & $\mathrm{R}^{2}$ & $\mathrm{RSME}$ \\
\hline $\mathrm{CP}$ & 20,07 & $1,97 \times 10^{-6}$ & 0,95 & 0,053 \\
$\mathrm{SP}$ & 28,66 & $2,92 \times 10^{-5}$ & 0,99 & 0,031 \\
\hline
\end{tabular}

Onde CP significa secagem com pré-tratamento por PVOD e SP, secagem sem PVOD

\section{CONCLUSÃO}

O modelo teórico de Fick apresentou bom ajuste aos dados experimentais, $\mathrm{R}^{2}$ médio de 0,99 . Isso sugere que a consideração de processo difusivo é adequada na secagem de yacon com ou sem o processo de desidratação osmótica (PVOD).

Os valores da difusividade efetiva aumentaram de acordo com a elevação da temperatura do ar de secagem, sendo maiores nas mostras tratadas osmoticamente. Este aumento da difusividade resultou em menores tempos de secagem nas amostras pré tratadas.

A dependência da difusividade em relação à temperatura foi descrita pela equação de Arrhenius, por meio da qual se obteve o valor da energia de ativação de 20,07 
e 28,66 $\mathrm{kJ} \mathrm{mol}^{-1}$ e RMSE de 0,053 e 0,031 respectivamente para as amostra com e sem o pré-tratamento.

\section{NOMENCLATURA}

$\begin{array}{ll}\mathrm{D}_{\text {eff }} & \text { Difusividade efetiva }\left[\mathrm{m}^{2} \mathrm{~s}^{-1}\right] \\ \mathrm{D}_{\mathrm{o}} & \text { Fator pré-exponencial }\left[\mathrm{m}^{2} \mathrm{~s}^{-1}\right] \\ \mathrm{E}_{\mathrm{a}} & \text { Energia de ativação }\left[\mathrm{kJ} \mathrm{mol}^{-1}\right. \\ \mathrm{T} & \text { Temperatura }\left[\mathrm{K} \mathrm{e} \mathrm{e}{ }^{\circ} \mathrm{C}\right] \\ \mathrm{MR} & \text { Razão de umidade }, \text { adimensi } \\ \mathrm{R} & \left.\mathrm{Constante} \mathrm{universal} \mathrm{dos} \mathrm{gases}^{-1} \mathrm{~mol}^{-1}\right] \\ \mathrm{t} & \text { Tempo }[\mathrm{s}] \\ \text { Subscritos } & \\ 0 & \text { Referente à condição inicial } \\ \mathrm{f} & \text { Referente à condição final } \\ \mathrm{eq} & \text { Equilíbrio }\end{array}$

\section{REFERÊNCIAS}

AOAC. Official methods of analysis of Association of official Analytical Chemists International: Gainsthersburg: Horwitz 2005.

CHAUHAN, O.; SINGH, A.; SINGH, A.; RAJU, P.; BAWA, A. Effects of osmotic agents on colour, textural, structural, thermal, and sensory properties of apple slices. International Journal of Food Properties, v.14, n.5, p.1037-104, 2011.

CORRÊA， J. L. G.; PEREIRA， L. M.; VIEIRA, G. S.; HUBINGER, M. D. Mass transfer kinetics of pulsed vacuum osmotic dehydration of guavas. Journal of Food Engineering, v.96, n.4, p.498-504, 2010.

CORRÊA, J. L. G.; ERNESTO, D. B.; ALVES, J. G. L. F.; ANDRADE, R. S. Optimisation of vacuum pulse osmotic dehydration of blanched pumpkin. International Journal of Food Science \& Technology, v.49, n.9, p.2008-2014, 2014.
CRANK, J. The mathematics of diffusion. Clarendon Press: Oxford. 1975.

DJENDOUBI MRAD, N.; BOUDHRIOUA, N.; KECHAOU, N.; COURTOIS, F.; BONAZZI, C. Influence of air drying temperature on kinetics, physicochemical properties, total phenolic content and ascorbic acid of pears. Food and Bioproducts Processing, v.90, n.3, p.433-441, 2012.

DOYMAZ, İ. Convective drying kinetics of strawberry. Chemical Engineering and Processing: Process Intensification, v. 47, n. 5, p. 914-919, 2008.

DOYMAZ, İ. Evaluation of some thin-layer drying models of persimmon slices (Diospyros kaki L.). Energy Conversion and Management, v.56, p.199-205, 2012.

DOYMAZ, İ.; DEMIR, H.; YILDIRIM, A. Drying of Quince Slices: Effect of Pretreatments on Drying and Rehydration Characteristics. Chemical Engineering Communications, v.202, n.10, p.1271-1279, 2014.

FANTE, C.; CORREAA, J.; NATIVIDADE, M.; LIMA, J.; LIMA, L. Drying of plums (Prunus sp, c.v Gulfblaze) treated with $\mathrm{KCl}$ in the field and subjected to pulsed vacuum osmotic dehydration. International Journal of Food Science \& Technology, v.46, n.5, p.1080-1085, 2011.

GIRI, S. K.; PRASAD, S. Drying kinetics and rehydration characteristics of microwavevacuum and convective hot-air dried mushrooms. Journal of Food Engineering, v.78, n.2, p.512-521, 2007.

GRAEFE, S.; HERMANN, M.; MANRIQUE, I.; GOLOMBEK, S.; BUERKERT, A. Effects of post-harvest treatments on the carbohydrate composition of yacon roots in the Peruvian 
Andes. Field Crops Research, v.86, n.23, p.157-165, 2004.

NOWACKA, M.; WIKTOR, A.; ŚLEDŹ, M.; JUREK, N.; WITROWA-RAJCHERT, D. Drying of ultrasound pretreated apple and its selected physical properties. Journal of Food Engineering, v.113, n.3, p.427-433, 2012.

OLIVEIRA, L. F. D. Desidratação osmotica com pulsos de vacuo e secagem a vacuo de yacon. (Tese Doutorado). Ciência dos Alimentos, Universidade Federal de Lavras, 2013.

PARK, S. H.; LAMSAL, B. P.; BALASUBRAMANIAM, V. 1 Principles of Food Processing. 2014.

SADEGHI, M.; MIRZABEIGI KESBI, O.; MIREEI, S. A. Mass transfer characteristics during convective, microwave and combined microwave-convective drying of lemon slices. Journal of the Science of Food and Agriculture, v.93, n.3, p.471-478, 2013.

SCHER, C. F.; RIOS, A. D. O.; NOREÑA, C. P. Z. Hot air drying of yacon (Smallanthus sonchifolius) and its effect on sugar concentrations. International Journal of Food Science \& Technology, v.44, n.11, p.2169-2175, 2009.

TAHERI-GARAVAND, A.; RAFIEE, S.; KEYHANI, A. Study on effective moisture diffusivity, activation energy and mathematical modeling of thin layer drying kinetics of bell pepper. Australian Journal of Crop Science, v.5, n.2, p.128-131, 2011.
VEGA-GÁLVEZ, A.; AH-HEN, K.; CHACANA, M.; VERGARA, J.; MARTÍNEZ-MONZÓ, J.; GARCÍASEGOVIA, P.; LEMUS-MONDACA, R.; DI SCALA, K. Effect of temperature and air velocity on drying kinetics, antioxidant capacity, total phenolic content, colour, texture and microstructure of apple (var. Granny Smith) slices. Food Chemistry, v.132, n.1, p.51-59, 2012.

WALKER, L.; SENADEERA, W. A variable diffusivity model for the drying of spherical food particulates. Applied Mechanics and Materials, v. 553, p. 94-99, 2014.

ZOGZAS, N. P.; MAROULIS, Z. B.; MARINOS-KOURIS, D. Moisture diffusivity data compilation in foodstuffs. Drying Technology, v.14, n.10, p.2225-2253, 1996.

ZOU, K., TENG, J., HUANG, L., DAI, X., \& WEI, B. Effect of osmotic pretreatment on quality of mango chips by explosion puffing drying. LWT-Food Science and Technology, v.51, n.1, p.253-259, 2013.

\section{AGRADECIMENTOS}

Ao Conselho Nacional de Pesquisa e Desenvolvimento Tecnológico - $\mathrm{CNPq}$, Coordenação de Aperfeiçoamento de Pessoal de Nível Superior-CAPES e Fundação de Amparo à Pesquisa do Estado de Minas Gerais - FAPEMIG, pelo apoio à pesquisa. 